\title{
Bi-Level Decentralized Active and Reactive Power Control for Large-Scale Wind Farm Cluster
}

Huang, Sheng; Wu, Qiuwei; Guo, Yifei; Lin, Zhongwei

Published in:

International Journal of Electrical Power \& Energy Systems

Link to article, DOI:

10.1016/j.ijepes.2019.03.045

Publication date:

2019

Document Version

Peer reviewed version

Link back to DTU Orbit

Citation (APA):

Huang, S., Wu, Q., Guo, Y., \& Lin, Z. (2019). Bi-Level Decentralized Active and Reactive Power Control for Large-Scale Wind Farm Cluster. International Journal of Electrical Power \& Energy Systems, 111, 201-215. https://doi.org/10.1016/j.ijepes.2019.03.045

\section{General rights}

Copyright and moral rights for the publications made accessible in the public portal are retained by the authors and/or other copyright owners and it is a condition of accessing publications that users recognise and abide by the legal requirements associated with these rights.

- Users may download and print one copy of any publication from the public portal for the purpose of private study or research.

- You may not further distribute the material or use it for any profit-making activity or commercial gain

- You may freely distribute the URL identifying the publication in the public portal 


\title{
Bi-Level Decentralized Active and Reactive Power Control for Large-Scale Wind Farm Cluster
}

\author{
Sheng Huang ${ }^{1}$, Qiuwei $\mathrm{Wu}^{1, *}$, Yifei Guo ${ }^{2}$, Zhongwei Lin $^{3}$ \\ 1.Center for Electric Power and Energy, Department of Electrical Engineering, Technical University of Denmark, Elektrovej 325, \\ Kgs. Lyngby, DK 2800 \\ 2.Key Laboratory of Power System Intelligent Dispatch and Control of Ministry of Education, School of Electrical Engineering, \\ Shandong University, Ji'nan 250061, People's Republic of China \\ 3.State Key Laboratory of Alternate Electrical Power System with Renewable Energy Sources, School of Control and Computer \\ Engineering, North China Electric Power University, Beijing 102206, People's Republic of China
}

\begin{abstract}
This paper proposes a bi-level decentralized active and reactive power control (DARPC) for the large-scale wind farm cluster (WFC) composed of several wind farms. The WFC tracks the active power reference from the transmission system operator (TSO) while controlling the bus voltage of the point of connection (POC), and maintaining the wind turbine (WT) terminal voltages stable in each wind farm. In the upper level, a distributed active and reactive power control scheme based on the consensus protocol is designed for the WFC, which can achieve fair active and reactive power sharing among multiple wind farms, and generates active and reactive power references for each wind farm. In the lower level, a centralized control scheme based on Model Predictive Control (MPC) is proposed, which can effectively regulates active and reactive power outputs of all WTs within the wind farm. The proposed centralized control scheme can maintain WTs terminal voltage close to the rated voltage while tracking the power reference from the upper level control. The DARPC can effectively reduce the computation burden of the WFC controller by distributing the computation and monitoring tasks to several wind farm controllers. Moreover, the communication cost is reduced. A WFC with 8 wind farms and totally $128 \mathrm{WT}$ s was used to validate the proposed DARPC scheme.
\end{abstract}

(C)

Keywords: consensus protocol, decentralized active and reactive power control, MPC, sensitivity calculation, wind farm cluster

\section{Introduction}

Renewable energy, especially wind energy, is developing rapidly over the world because of the pressure of reducing carbon emission and dependence on fossil fuels. The European Wind Energy Association (EWEA) estimates that the installed capacity of wind power could expand to $320 \mathrm{GW}$ by 2030 [1]. In Denmark, the target is to achieve $50 \%$ electricity from wind by 2020 and become $100 \%$ fossil fuel free by 2050 [2].

\footnotetext{
$1 *$ Corresponding author: Qiuwei.Wu

Email: qw@elektro.dtu.dk
} 
As wind power penetration increases, the large-scale wind farm cluster (WFC) is required to have the same level of performance as conventional generation plants [3]. Due to the intermittency of wind power, the increasing wind power penetration has introduced various challenges to the power system operation [4]. Such challenges include power reference tracking, voltage regulation, ancillary services for power systems, etc. [5].

Usually, the short circuit ratio at the point of connection (POC) is small because large-scale wind farms are mainly located in areas far from load centers [6], and the grid at the POC is weak. The voltage fluctuation caused by the wind power variation is quite large. Voltage support at the POC has been specified in several grid codes around the world. The WFC controller receives the power dispatch command and specific technical requirement from the transmission system operator (TSO), such as the POC voltage requirement and reactive power dispatch command [7].

For active power control of the large-scale wind farms, the main control objective is power tracking, and the control strategy can be classified into proportional distribution (PD) control [8], [9], proportionalintegral (PI) control [10] and optimal active power control [11], [12]. Among these, the PD strategy is widely adopted in modern wind farms due to its simple implementation, and considers the available power and Var capability of wind turbines (WTs) [13], [14]. The voltage and reactive power control of wind farms, as one of the major topics of wind power integration, have motivated a great number of studies. In the conventional control strategy, wind farms provide a certain level reactive power support at POC [15], [16], or regulate the power factor at the POC [17], [18], using the slope-based function or the proportional integral (PI) controller to obtain the reactive power reference [19]. For the optimization-based control strategies of wind farm, reference [20] proposed a coordinated voltage control scheme based on a PI controller to generate reactive power references for the wind farm clusters. The voltage reference for the pilot bus is determined by an optimization algorithm to minimize active power losses in the wind farm grid. In [21], a hierarchical automatic voltage control system to support wind power integration was designed to optimize the voltage and reactive power distribution inside the wind farm. Reference [22] proposed an autonomous wind farm voltage controller based on model predictive control(MPC), which aims to maintain all the bus voltages in wind farm within their feasible range and maximize the fast dynamic Var reserve, and consider various reactive power regulation devices, such as static Var compensators (SVC), static Var generators (SVG), on load tap changing (OLTC) Transformer, etc. References [23], [24] proposed a enhanced voltage control strategy based on MPC for offshore wind farms. This strategy aims to optimize and maintain voltages within the feasible range and reduce system power losses.

According to the previous studies, most of the control strategies are centralized control, which receives operation information from all WTs and sends the power reference to them. For the large-scale WFC with several wind farms, and each wind farm consists of hundreds of WTs, the computation burden of the central controller is quite heavy. In addition, the cost of communication networks is high, and the control performance largely depends on the central controller, implying low robustness.

Therefore, a bi-level decentralized active and reactive power control (DARPC) scheme for the largescale WFC which integrates the centralized control and distributed control is proposed in this paper. The upper level control is designed in a distributed manner based on the consensus protocol, which aims to track active power dispatch command from the TSO, and regulate reactive power of each wind farm to control the POC voltage in a feasible range. Besides, the discrete variables, such as OLTC tap position, are also considered. At this level, the wind farm controllers only exchange information with its neighbors, removing the requirement of a central supervisor controller. As such, each controller can control the active and reactive power without global information of the WFC. The lower level adopts a centralized control, which is based on MPC. Each wind farm controller receives the active and reactive power references from the upper-level, and controls active and reactive power of WTs in the wind farm, not only tracking the power reference, but also controlling all WT terminal voltages within the feasible range.

The main contribution of this paper is the bi-level DARPC strategy which is suitable for the large-scale WFC. The active and reactive power control is optimally coordinated to track the dispatch command, and keep the POC voltage in a feasible range. The computation and monitoring tasks of the WFC controller are distributed to several wind farm controllers, and then the control problem can be solved in parallel. In the upper-level control, the consensus protocol method is used to obtain the active and reactive power 
references for each wind farm in a more efficient and fast way. Compared with the centralized control, since each wind farm only exchanges information with its neighbors, the communication cost is reduced. Moreover, the WFC controller has good plug and-play capability, providing the convenience to extend it. In the lower-level control, since the MPC algorithm can take into account the dynamic response of the system , and optimize the multi-input multi-output problem, the impacts of active and reactive power of WTs on voltage variations are also taken into consideration to improve the voltage control performance. With the MPC, the wind farm can accurately track the power dispatch command, and minimize the voltage deviation for each WT terminal.

The paper is organized as follows. Section 2 gives an overview of the proposed bi-level control scheme. The upper level distributed control based on the consensus protocol is presented in Section 3. The lower level centralized control based on the MPC is described in Section 4. Simulation results are presented and discussed in Section 5, followed by conclusions.

\section{Bi-level control architecture}

\subsection{Configuration of a WFC}

Fig. 1 shows the typical configuration of a WFC, which is connected to the external $380 \mathrm{kV}$ AC grid through a $20 \mathrm{~km} 380 \mathrm{kV}$ transmission cable with the nominal power rating of $800 \mathrm{MW}$. The OLTC is at the HV/MV transformer of the main substation of the WFC. The WFC consists of eight wind farms, each with $16 \times 6.25 \mathrm{MW}$ WTs. Eight WTs are connected by a $33 \mathrm{kV}$ feeder and placed with a distance of $4 \mathrm{~km}$. There are eight collector substations, which are connected to the POC bus through $155 \mathrm{kV}$ transmission cables.

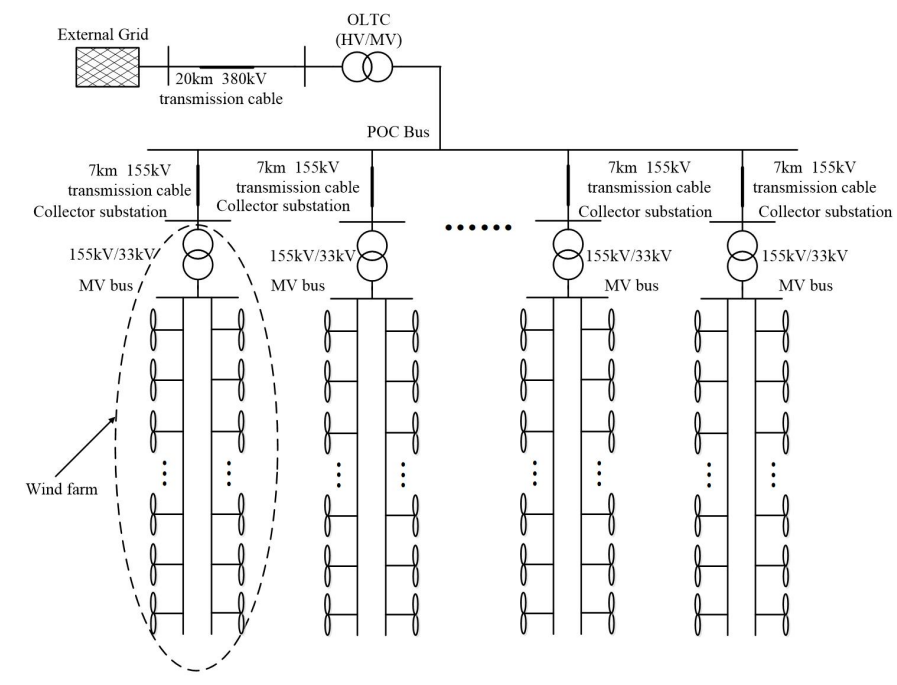

Fig. 1. configuration of WFC.

\subsection{Concept of the bi-level DARPC}

The structure of the proposed bi-level DARPC scheme is shown in Fig. 2. In the WFC, each wind farm is equipped with a wind farm controller consisting of two levels, i.e., the upper-level control and lower-level control.

The upper-level control is designed in a distributed manner based on the consensus protocol. The wind farm controllers within the WFC with direct access to the power reference and POC voltage reference from the TSO are defined as leaders, and others are defined as followers. Moreover, the leader controllers receive the information including tap position, action of OLTC, and POC voltage measurement. The upper-level control aims to achieve fair active and reactive power sharing among multiple wind farms according to their 
available power. In this scheme, wind farm controllers only exchange information with their neighboring controllers. In the lower-level control, a centralized active and reactive power control scheme is used based on the MPC, in which each wind farm controller optimally coordinates all WTs inside the wind farm to track the active and reactive power reference from the upper level control, and minimizes the voltage deviation of each WT terminal.

The communication network topology is shown in Fig. 3, which is designed in a redundant way, and meets the " $N-1$ " principle, i.e., the communication network graph will still be a connected graph despite a communication link failure between any two controllers.

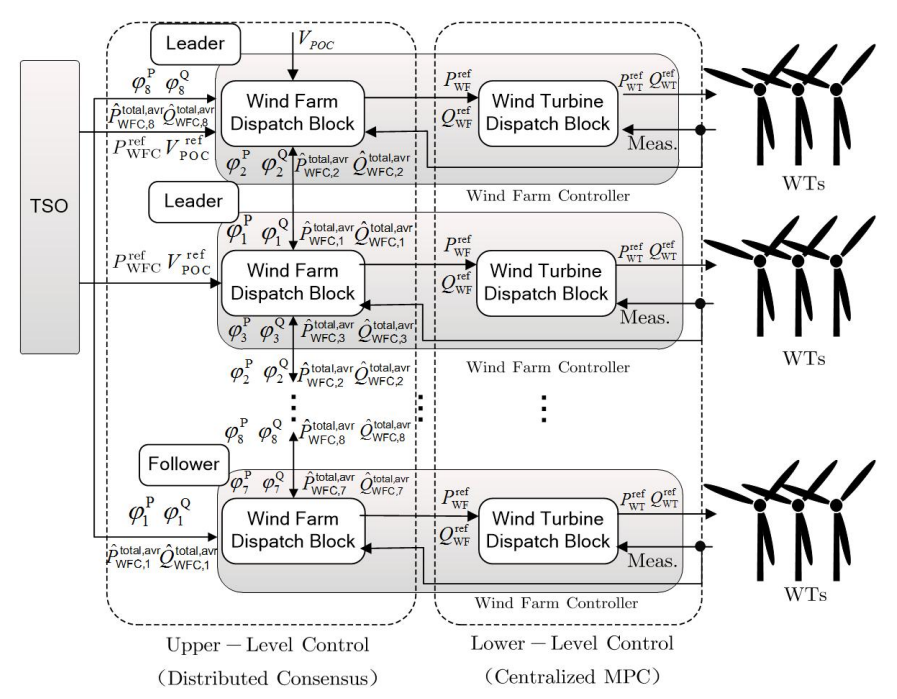

Fig. 2. Control structure.

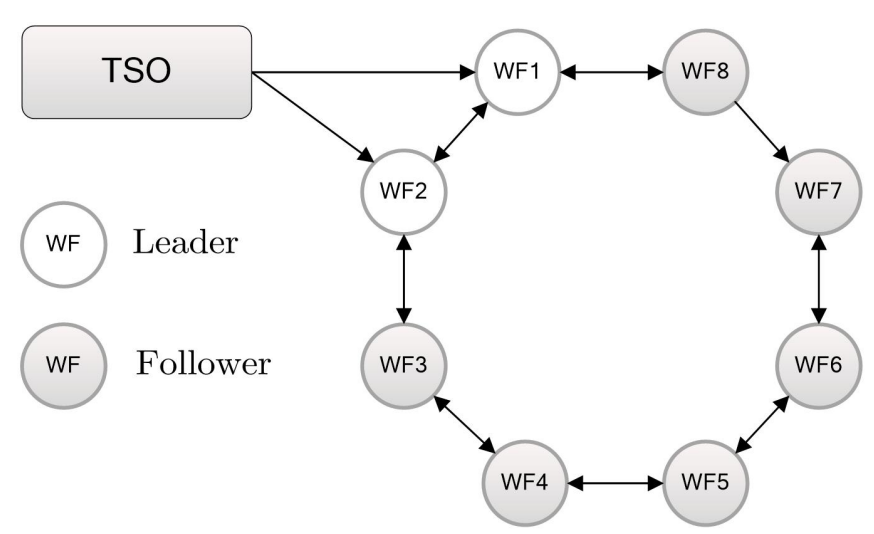

Fig. 3. communication network.

\section{Consensus-based distributed active power and reactive power dispatch for WFC}

\subsection{Graph theory}

Consider a digraph $\mathcal{D}=(\mathcal{V}, \xi)$, where $\mathcal{V}=(1,2, \ldots, N)$ denotes the set of distributed nodes. $\xi \subseteq \mathcal{V} \times \mathcal{V}$ denotes the directed paths, and an adjacency matrix $\mathcal{A}=\left[a_{i j}\right]$ with nonnegative adjacency elements $a_{i j}$. If there exists a directed path from node $i$ to node $j,(i, j) \in \xi$, it is defined as node $i$ is adjacent to node $j$. All 
paths in digraph $\mathcal{D}$ are connected nodes bidirectionally, which is defined as strongly connected. Without loss of any generality, the adjacency matrix $\mathcal{A}(\mathcal{D})$ can be expressed as,

$$
[\mathcal{A}(\mathcal{D})]_{i j}= \begin{cases}1, & \text { if }(i, j) \in \xi \\ 0, & \text { otherwise }\end{cases}
$$

\subsection{Distributed active power control of WFC}

For distributed active control, wind farm controllers of the WFC only exchange information with their immediate neighbors to reduce the communication requirement, implying none of wind farms directly knows the total available power of the cluster. Therefore, the upper-level controller must synchronize the total available power.

The available power of a wind farm, denoted as $P_{\mathrm{WF}, i}^{\mathrm{avi}}$, is the sum of maximum available power of all WTs inside the wind farm. It can be obtained through the operation information gathered from each WT, which is expressed by,

$$
P_{\mathrm{WF}, i}^{\mathrm{avi}}=\sum_{j=1}^{\left|\mathcal{N}_{\mathrm{T}}^{(i)}\right|} P_{\mathrm{WT}, j}^{\mathrm{avi}}, \quad \forall i \in \mathcal{N}_{\mathrm{F}}
$$

where $\mathcal{N}_{\mathrm{F}}$ and $\mathcal{N}_{\mathrm{T}}^{(i)}$ denote the wind farm set and WT set of the wind farm $i$, respectively, and $P_{\mathrm{WT}, j}^{\text {avi }}$ is the maximum available power of WT- $j$. In a control period, the wind speed $v_{\mathrm{m}}$ can be measured by each WT. The maximum power coefficient $C_{\mathrm{P}}^{\max }$ and optimal tip speed ratio $\lambda_{\mathrm{opt}}$ are known to each WT controller. The available wind power of each WT can be expressed as,

$$
P_{\mathrm{WT}}^{\mathrm{avi}}=\frac{1}{2} \rho A C_{\mathrm{P}}^{\max }\left(\lambda_{\mathrm{opt}}, \beta\right) v_{\mathrm{m}}^{3}
$$

where $\rho$ is the air density, $A$ is the WT swept area, and $\beta$ is the pitch angle. Then the total available active power of the WFC $P_{\mathrm{WFC}}^{\text {total,avi }}$ can be calculated by,

$$
P_{\mathrm{WFC}}^{\mathrm{total}, \mathrm{avi}}=\sum_{i=1}^{\left|\mathcal{N}_{\mathrm{F}}\right|} P_{\mathrm{WF}, i}^{\mathrm{avi}}
$$

Since wind farm controllers only exchange information with their immediate neighbors, none of wind farms directly knows the total available power of the WFC. Based on the consensus protocol, the average active power of each wind farm can be obtained as follows,

$$
\dot{\hat{P}}_{\mathrm{WF}, i}^{\mathrm{total}, \mathrm{avr}}=\mu_{\mathrm{P}} \sum_{j=1}^{\left|\mathcal{N}_{\mathrm{F}}\right|} a_{i j}\left[\hat{P}_{\mathrm{WF}, j}^{\mathrm{total}, \mathrm{avr}}(t)-\hat{P}_{\mathrm{WF}, i}^{\mathrm{total}, \mathrm{avr}}(t)\right]
$$

where $\hat{P}_{\mathrm{WF}, i}^{\mathrm{total}, \mathrm{avr}}$ is the estimated average active power of $i$ th wind farm, $\mu_{\mathrm{P}}$ is a constant gain, and $a_{i j}$ is entries form the adjacency matrix $\mathcal{A}(\mathcal{D})$. Denotes the total available wind power as $P_{\mathrm{WFC}}^{\text {total,avi }}$. The average available power of the cluster estimated at each wind farm will converge to $P_{\mathrm{WFC}}^{\mathrm{total}, \mathrm{avi}} /\left|\mathcal{N}_{\mathrm{F}}\right|$ in finite time. The proof of convergence can be found in [25]. Then, the total available power of the WFC $\hat{P}_{\mathrm{WFC}}^{\text {total,avi }}$ can be estimated by each wind farm controller as,

$$
\hat{P}_{\mathrm{WFC}}^{\mathrm{total}, \text { avi }}=\left|\mathcal{N}_{\mathrm{F}}\right| \cdot \hat{P}_{\mathrm{WF}, i}^{\mathrm{total}, \mathrm{avr}}, \forall i \in \mathcal{N}_{\mathrm{F}}
$$

The estimation of the total available power of the WFC is executed every several seconds. The convergence time shall be much shorter than the execution period. Define the active power utilization ratio of a wind farm as $\varphi_{i} \triangleq P_{\mathrm{WF}, i}^{\mathrm{ref}} / P_{\mathrm{WF}, i}^{\mathrm{avi}}, \forall i \in \mathcal{N}_{\mathrm{F}}$, the aim of the distributed active power control is to achieve consensus utilization ratio sharing among multiple wind farms, such that,

$$
\varphi_{1}^{\mathrm{P}}=\varphi_{2}^{\mathrm{P}}=\cdots=\varphi_{\left|\mathcal{N}_{\mathrm{F}}\right|}^{\mathrm{P}}=\varphi_{\mathrm{P}}^{*}
$$


where $\varphi_{i}^{\mathrm{P}}$ is the utilization ratio of the $i$ th wind power, and $\varphi_{\mathrm{P}}^{*}$ is the desired optimal utilization ratio. Define the leaders and followers sets as $\mathcal{L}$ and $\mathcal{F}$, respectively. For the leaders, who directly receive power reference of the cluster from the TSO, their active power control law is designed as,

$$
\varphi_{i}^{\mathrm{P}}(t)=\left\{\begin{array}{ccc}
\frac{P_{\mathrm{WFC}}^{\mathrm{ref}}}{\hat{P}_{\mathrm{WFC}}^{\mathrm{tota}, a v i}}, & \text { if } \quad P_{\mathrm{WFC}}^{\mathrm{ref}} \leq \hat{P}_{\mathrm{WFC}}^{\mathrm{total}, \text { avi }}, \\
1, & \text { if } \quad P_{\mathrm{WFC}}^{\mathrm{ref}}>\hat{P}_{\mathrm{WFC}}^{\text {total,avi }},
\end{array} \quad \forall i \in \mathcal{L} .\right.
$$

where $P_{\text {WFC }}^{\text {ref }}$ is the active power reference of the WFC. For the followers, they track the utilization ratio set by the leaders using the averaging consensus protocol, which is,

$$
\dot{\varphi}_{i}^{\mathrm{P}}(t)=\varepsilon_{\mathrm{P}} \sum_{j=1}^{\left|\mathcal{N}_{\mathrm{F}}\right|} a_{i j}\left[\varphi_{j}^{\mathrm{P}}(t)-\varphi_{i}^{\mathrm{P}}(t)\right], \quad \forall i \in \mathcal{F}
$$

where $\varepsilon_{\mathrm{P}}$ is a constant gain, which affects the convergence. Once the optimal utilization ratio is obtained, the power reference $P_{\mathrm{WF}, i}^{\mathrm{ref}}$ for each wind farm can be given by,

$$
P_{\mathrm{WF}, i}^{\mathrm{ref}}=\varphi_{i}^{\mathrm{P}} \cdot P_{\mathrm{WF}, i}^{\mathrm{avi}}, \quad \forall i \in \mathcal{N}_{\mathrm{F}}
$$

where $P_{\mathrm{WF}, i}^{\mathrm{ref}}$ is the power reference of $i$ th wind farm.

\subsection{Distributed reactive power control of WFC}

\subsubsection{Equivalent model of WFC}

In a large-scale WFC, each wind farm is connected to the POC bus. Since the active power output of each wind farm is decoupled, each wind farm can be represented by a current source and equivalent impedance. Based on the analytical approach in [26], the equivalent representation of a large-scale WFC system is derived, which is shown in Fig. 4.

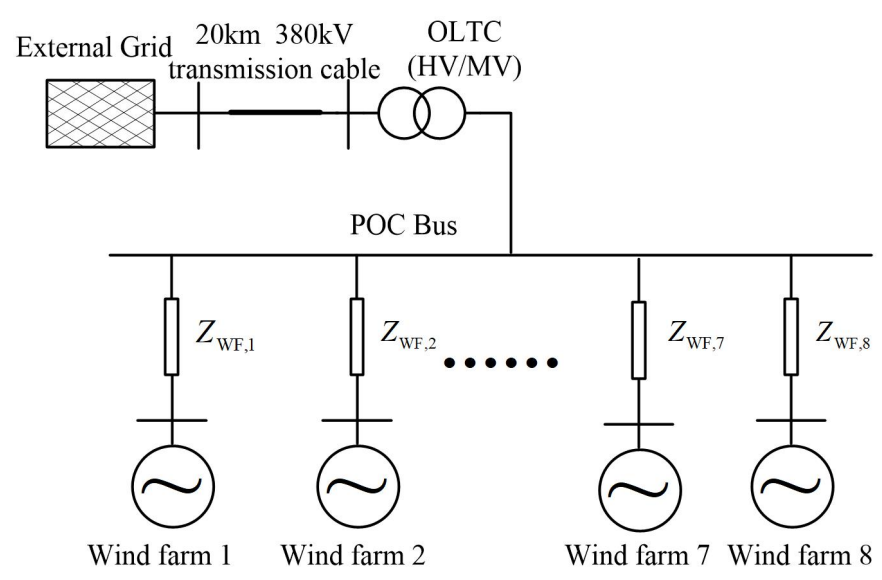

Fig. 4. The equivalent configuration of WFC.

Based on Fig. 4, the equivalent impedance of each wind farm can be expressed as,

$$
Z_{\mathrm{WF}, i}=\frac{2 \sum_{m=1}^{\left|\mathcal{N}_{\mathrm{T}}^{i}\right| / 2} m^{2} Z_{\mathrm{m}}}{\left|\mathcal{N}_{\mathrm{T}}\right|^{2}}
$$

where $Z_{\mathrm{m}}$ is the cable impedance between the two adjacent WTs, $m$ denotes the number of cables inside the wind farm. 
Define the vectors $V(k)=\left[V_{1}(k), V_{2}(k), \ldots, V_{\mathcal{N}_{\mathrm{d}}}(k)\right]^{\mathrm{T}}, \theta(k)=\left[\theta_{1}(k), \theta_{2}(k), \ldots, \theta_{\mathcal{N}_{\mathrm{d}}}(k)\right]^{\mathrm{T}}$ as voltage and angle of equivalent current source at period k, respectively. $\mathcal{N}_{\mathrm{d}}$ denotes the node set of the WFC. Vectors $P(k)=\left[P_{1}(k), P_{2}(k), \ldots, P_{\mathcal{N}_{\mathrm{F}}}(k)\right]^{\mathrm{T}}$ and $Q(k)=\left[Q_{1}(k), Q_{2}(k), \ldots, Q_{\mathcal{N}_{\mathrm{F}}}(k)\right]^{\mathrm{T}}$ are active and reactive power output, respectively. Then the increment model of the WFC can be expressed as ,

$$
\left[\begin{array}{c}
\Delta P(k) \\
\Delta Q(k)
\end{array}\right]=\left[\begin{array}{cc}
H & N \\
K & L
\end{array}\right]\left[\begin{array}{c}
\Delta \theta(k) \\
\Delta V(k)
\end{array}\right]
$$

where $H=\left[\frac{\partial P}{\partial \theta}\right], N=\left[\frac{\partial P}{\partial V}\right], K=\left[\frac{\partial Q}{\partial \theta}\right], L=\left[\frac{\partial Q}{\partial V}\right]$. Then we obtain,

$$
\begin{gathered}
\Delta \theta(k)=-H^{-1} N \Delta V(k)+H^{-1} \Delta P(k) \\
\Delta V(k)=\left(L-K H^{-1} N\right)^{-1}\left(\Delta Q(k)-K H^{-1} \Delta P(k)\right)=S \Delta Q(k)+C \Delta P(k)
\end{gathered}
$$

where $C=-\left(\left(L-K H^{-1} N\right)^{-1}\right) K H^{-1}$. The entries of matrixes $S, C$ are related to the sensitivity coefficients $\left[\frac{\partial \theta}{\partial P}\right],\left[\frac{\partial V}{\partial P}\right],\left[\frac{\partial \theta}{\partial Q}\right]$, and $\left[\frac{\partial V}{\partial Q}\right]$. The calculation of sensitivity coefficients is presented in detail in Subsection 4.2. In this paper, it is assumed the WFC has a fixed electrical network configuration, and the sensitivity coefficients can be calculated offline. Moreover, since the reactive power calculation is after the active power calculation, $C$ can be seen as a constant. In the upper level, each wind farm provides reactive power to maintain the POC bus voltage at the rated value while tracking the active power dispatch command. The variation of the POC voltage $\Delta V_{\mathrm{POC}}$ can be expressed as,

$$
\Delta V_{\mathrm{POC}}(k)=S_{\mathrm{POC}} \Delta Q(k)+C_{\mathrm{POC}} \Delta P(k)
$$

where $S_{\mathrm{POC}}, C_{\mathrm{POC}}$ are the matrixes which describe the relationship between the POC bus voltage and output reactive and active power from each wind farm. The entries of $S_{\mathrm{POC}}, C_{\mathrm{POC}}$ are obtained from matrixes $S, C$, which are defined by the sensitivity coefficients $\left[\frac{\partial \theta_{\mathrm{POC}}}{\partial P}\right],\left[\frac{\partial V_{\mathrm{POC}}}{\partial P}\right],\left[\frac{\partial \theta_{\mathrm{OPC}}}{\partial Q}\right]$, and $\left[\frac{\partial V_{\mathrm{POC}}}{\partial Q}\right]$. When active and reactive power output is known, the voltage and angle of the equivalent current source can be obtained by power flow calculation.

\subsubsection{Reactive power reference calculation with OLTC}

For the reactive power control, the leader controller has access to the upper level control, which provides voltage information from the POC bus and OLTC. As such, the leader controller can calculate the required total reactive power of the WFC to realize the desired control objective. An integral controller can be used by the leader controller to update the reactive power reference as follows,

$$
\Delta Q_{\mathrm{WFC}}=\alpha\left(V_{\mathrm{POC}}^{\mathrm{ref}}-V_{\mathrm{POC}}\right)
$$

where $V_{\mathrm{POC}}^{\text {ref }}$ is the POC voltage reference, $\Delta Q_{\mathrm{WFC}}$ is the WFC incremental reactive power reference, and $\alpha>0$ is a coefficient. In this paper, it updates every 5 seconds. When a disturbance occurs at the grid side or the WFC side, the OLTC will change the tap position to maintain the POC voltage in a feasible range. So the integral controller should consider the OLTC action when updating the reactive power reference. The OLTC working principle is illustrated in Fig.5.

The details of the OLTC working principle can be found in [22], [27]. $V_{\mathrm{BD}}$ is the deadband to avoid unnecessary switching around the reference voltage. At time $t=t_{\text {tri }}$, the POC voltage variation exceeds the deadband, and a timer is triggered, the tap $n_{\text {tap }}$ will increase $n_{\text {tap }}+1$ or $n_{\text {tap }}-1$ after time delay $T_{\text {delay. }}$. In order to obtain the reactive power reference accurately, for each control step, the leader controller should check if there is a potential tap action within the control period. From (15), the leader controller should update the reactive power reference as follows,

$$
\Delta Q_{\mathrm{WFC}}=\left\{\begin{array}{lc}
\alpha\left(V_{\mathrm{POC}}^{\mathrm{ref}}-V_{\mathrm{POC}} \pm \Delta V_{\mathrm{tap}}\right), & \text { if } t_{n}<t_{\text {act }}<t_{n+1} ; \\
\alpha\left(V_{\mathrm{POC}}^{\text {ref }}-V_{\mathrm{POC}}\right), & \text { otherwise. }
\end{array}\right.
$$

where $\Delta V_{\text {tap }}$ is the voltage change per tap. Since the coefficient $\alpha$ affects the variation of reactive power reference of the WFC in every control period, it must be chosen to ensure the stability of the closed-loop 


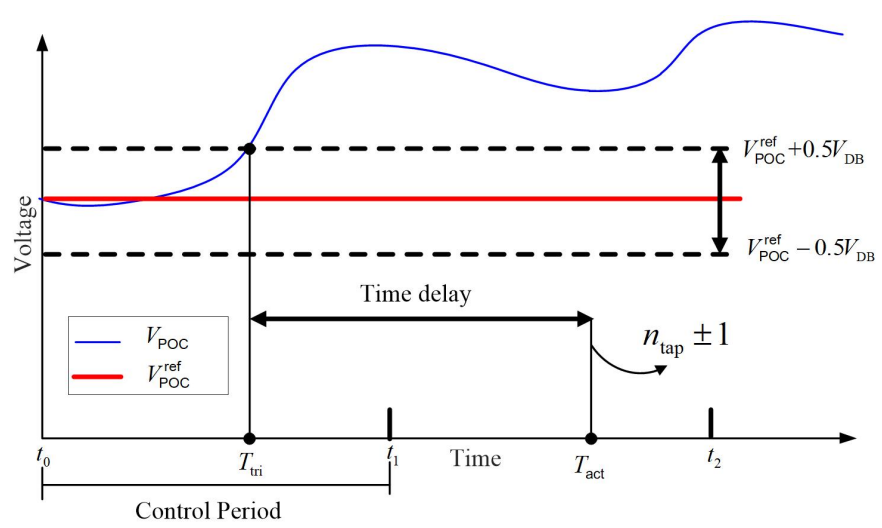

Fig. 5. Working principle of OLTC.

system. When the leader controller obtains the reactive power reference, the reactive power reference is shared by wind farms. In order to achieve fair reactive power sharing among multiple wind farms, the reactive power utilization ratio should be fair. Thus, we can get,

$$
\Delta Q(k)=E \Delta Q_{\mathrm{WFC}}(k)=E \alpha\left(V_{\mathrm{POC}}^{\mathrm{ref}}-V_{\mathrm{POC}} \pm \Delta V_{\mathrm{tap}}\right)
$$

where $E=\left[\beta_{1}, \beta_{2}, \ldots, \beta_{\mathcal{N}_{\mathrm{F}}}\right]^{\mathrm{T}} . \beta_{i}$ is the ratio of the available reactive power of $i$ th wind farm over the WFC reactive power. It can be obtained by,

$$
\beta_{i}=\frac{\sqrt{S_{\mathrm{WF}, i}^{2}-P_{\mathrm{WF}, L}^{2}}}{\sum_{i=1}^{\mathcal{N}_{\mathrm{F}}} \sqrt{S_{\mathrm{WF}, i}^{2}-P_{\mathrm{WF}, L}^{2}}}
$$

where $S_{\mathrm{WF}, i}$ is the rated capacity of $i$ th wind farm. Since $V_{\mathrm{POC}}(k)$ and $\Delta V_{\text {tap }}$ are known in every control period, $\Delta V_{\text {tap }}$ can be expressed by,

$$
\Delta V_{\text {tap }}=\delta V_{\mathrm{POC}}(k)
$$

where $\delta$ is a coefficient. The discrete time relation that describes how the POC voltage magnitudes evolve with time can be expressed as,

$$
V_{\mathrm{POC}}(k+1)=V_{\mathrm{POC}}(k)+S_{\mathrm{POC}} \Delta Q(k)+C_{\mathrm{POC}} \Delta P(k)
$$

Then, by substituting (17) into (20), we get can get,

$$
\left.V_{\mathrm{POC}}(k+1)=\left[1-(1 \pm \delta) \alpha S_{\mathrm{POC}} E\right)\right] V_{\mathrm{POC}}(k)+\alpha S_{\mathrm{POC}} E V_{\mathrm{POC}}^{\mathrm{ref}}(k)+C_{\mathrm{POC}} \Delta P(k)
$$

In this paper, $V_{\mathrm{POC}}^{\mathrm{ref}}(k)$ is constant, which is set as 1 p.u., and $C_{\mathrm{POC}} \Delta P(k)$ is bounded in every control period, which are related to the capacity of each wind farm. Since the system is a linear time-invariant system driven by bounded inputs $V_{\mathrm{POC}}^{\mathrm{ref}}(k)$ and $C_{\mathrm{POC}}(k) \Delta P(k)$. $V_{\mathrm{POC}}(k)$ should be bounded for all $\mathrm{k}$ to ensure the stability of this system. In order to ensure that the system is asymptotically stable, $\alpha$ must be bounded by,

$$
\left.\mid\left[1-(1 \pm \delta) \alpha S_{\mathrm{POC}} E\right)\right] \mid<1
$$

\subsubsection{Reactive power control based on consensus protocol}

In this subsection, a distributed reactive power dispatch scheme is designed to regulate reactive power among multiple wind farms inside the WFC. The estimation of the total available reactive power of the WFC is executed after the active power calculation. Once each wind farm gets the active power reference, the available reactive power of the wind farm, denoted as $Q_{\mathrm{WF}, i}^{\mathrm{avi}}$, can be expressed as [20],

$$
Q_{\mathrm{WF}, i}^{\mathrm{avi}}=\sqrt{S_{\mathrm{WF}, i}^{2}-P_{\mathrm{WF}, i}^{2}}
$$


Then the total available reactive power of the WFC can be calculated by,

$$
Q_{\mathrm{WFC}}^{\mathrm{total,avi}}=\sum_{i=1}^{\left|\mathcal{N}_{\mathrm{F}}\right|} Q_{\mathrm{WF}, i}^{\mathrm{avi}}
$$

A distributed estimator based on the distributed averaging consensus is designed to synchronize the total available reactive power as follows,

$$
\dot{\hat{Q}}_{\mathrm{WF}, i}^{\text {total,avr }}=\mu_{\mathrm{Q}} \sum_{j=1}^{\left|\mathcal{N}_{\mathrm{F}}\right|} a_{i j}\left[\hat{Q}_{\mathrm{WF}, j}^{\text {total,avr }}(t)-\hat{Q}_{\mathrm{WF}, i}^{\text {total,avr }}(t)\right]
$$

where $\hat{Q}_{\mathrm{WF}, i}^{\mathrm{total}, \mathrm{avr}}$ is estimated the average reactive power of $i$ th wind farm, and $\mu_{\mathrm{Q}}$ is a constant gain. The total available reactive power of the WFC can be estimated by each wind farm controller as,

$$
\hat{Q}_{\mathrm{WFC}}^{\text {total,avi }}=\left|\mathcal{N}_{\mathrm{F}}\right| \cdot \hat{Q}_{\mathrm{WF}, i}^{\text {total,avr }}, \forall i \in \mathcal{N}_{\mathrm{F}} .
$$

The aim of the upper level for reactive power control is to ensure that each of the wind farm is treated fairly according to their available reactive power, which means that every wind farm should operate with a common reactive power utilization ratio. It can be expressed by,

$$
\varphi_{1}^{Q}=\varphi_{2}^{Q}=\cdots=\varphi_{\left|\mathcal{N}_{\mathrm{F}}\right|}^{Q}=\varphi_{\mathrm{Q}}^{*}
$$

where $\varphi^{Q}$ is the utilization ratio of reactive power, and $\varphi_{\mathrm{Q}}^{*}$ is the desired optimal utilization ratio. For the leaders, their reactive power control law is designed as,

$$
\varphi_{i}(t)=\left\{\begin{array}{ccc}
\frac{Q_{\mathrm{WFC}}^{\text {ref }}}{\hat{Q}_{\mathrm{WFC}}^{\text {totav }},}, & \text { if } & \left|Q_{\mathrm{WFC}}^{\mathrm{ref}}\right| \leq\left|\hat{Q}_{\mathrm{WFC}}^{\text {total, avi }}\right|, \quad \\
1, & \text { if } & \left|Q_{\mathrm{WFC}}^{\text {ref }}\right|>\left|\hat{Q}_{\mathrm{WFC}}^{\text {total,avi }}\right|,
\end{array} \quad \forall i \in \mathcal{L} .\right.
$$

where $Q_{\mathrm{WFC}}^{\mathrm{ref}}$ is the reactive reference for the WFC. For the followers, they track the utilization ratio set by the leaders using the averaging consensus protocol, which is,

$$
\dot{\varphi}_{i}^{\mathrm{Q}}(t)=\varepsilon_{\mathrm{Q}} \sum_{j=1}^{\left|\mathcal{N}_{\mathrm{F}}\right|} a_{i j}\left[\varphi_{j}^{\mathrm{Q}}(t)-\varphi_{i}^{\mathrm{Q}}(t)\right], \quad \forall i \in \mathcal{F}
$$

where $\varepsilon_{\mathrm{Q}}$ is a constant gain, which affects the convergence. Once the optimal utilization ratio is obtained, the power reference $Q_{\mathrm{WF}, i}^{\mathrm{ref}}$ for each wind farm can be given by,

$$
Q_{\mathrm{WF}, i}^{\mathrm{ref}}=\varphi_{i}^{Q} \cdot Q_{\mathrm{WF}, i}^{\mathrm{avi}}, \forall i \in \mathcal{N}_{\mathrm{F}}
$$

\section{Centralized MPC-based avtive and reactive power control of wind farm}

In the lower-level control, the wind farm controller regulates the active and reactive power outputs of all WTs inside the wind farm to track the power reference from the upper level control, and maintain all WT terminal voltages in a feasible range. Since modern WTs are equipped with power electronic converters, the response is quite fast.

\subsection{Modeling of wind farm}

Suppose the active and reactive power references and measurements of the WT are $P_{\mathrm{wt}}^{\mathrm{ref}}, Q_{\mathrm{wt}}^{\mathrm{ref}}, P_{\mathrm{wt}}^{0}$, and $Q_{\mathrm{wt}}^{0}$. Considering the time delay of the communication system and dynamic response of the WT control system, if all the WTs are controlled with active and reactive power control mode, the following turbine 
transfer function can be obtained, in which the dynamic behavior of the power control loops of WTs could be described by a first-order lag function,

$$
\begin{aligned}
\Delta \dot{P}_{\mathrm{wt}} & =\frac{1}{1+s T_{\mathrm{wt}}^{\mathrm{P}}} \Delta P_{\mathrm{wt}}^{\mathrm{ref}} \\
\Delta \dot{Q}_{\mathrm{wt}} & =\frac{1}{1+s T_{\mathrm{wt}}^{\mathrm{Q}}} \Delta Q_{\mathrm{wt}}^{\mathrm{ref}}
\end{aligned}
$$

where $T_{\mathrm{wt}}^{\mathrm{P}}$ and $T_{\mathrm{wt}}^{\mathrm{P}}$ are time constant. Accordingly, the continuous state space model of a wind farm with $N_{\mathrm{w}}$ WTs can be formulated as,

$$
\Delta \dot{x}=\boldsymbol{A} \Delta x+\boldsymbol{B} \Delta u
$$

where

$$
\begin{gathered}
\Delta x=\left[\Delta P_{\mathrm{wt}, 1}, \ldots, \Delta P_{\mathrm{wt}, \mathcal{N}_{\mathrm{T}}}, \Delta Q_{\mathrm{wt}, 1}, \ldots, \Delta Q_{\mathrm{wt}, \mathcal{N}_{\mathrm{T}}}\right] \\
\Delta u=\left[\Delta P_{\mathrm{wt}, 1}^{\mathrm{ref}}, \ldots, \Delta P_{\mathrm{wt}, \mathcal{N}_{\mathrm{T}}}^{\mathrm{ref}}, \Delta Q_{\mathrm{wt}, 1}^{\mathrm{ref}}, \ldots, \Delta Q_{\mathrm{wt}, \mathcal{N}_{\mathrm{T}}}^{\mathrm{ref}}\right] \\
\boldsymbol{A}=\left[\begin{array}{cc}
A_{\mathrm{wt}}^{\mathrm{P}} & 0 \\
0 & A_{\mathrm{wt}}^{\mathrm{Q}}
\end{array}\right] \boldsymbol{B}=\left[\begin{array}{cc}
B_{\mathrm{wt}}^{\mathrm{P}} & 0 \\
0 & B_{\mathrm{wt}}^{\mathrm{Q}}
\end{array}\right] \\
A_{\mathrm{wt}}^{\mathrm{P}}=\operatorname{diag}\left[-1 / T_{\mathrm{wt}, 1}^{\mathrm{P}},-1 / T_{\mathrm{wt}, 2}^{\mathrm{P}}, \ldots,-1 / T_{\mathrm{wt}, \mathcal{N}_{\mathrm{T}}}^{\mathrm{Q}}\right], \\
B_{\mathrm{wt}}^{\mathrm{P}}=\operatorname{diag}\left[1 / T_{\mathrm{wt}, 1}^{\mathrm{P}}, 1 / T_{\mathrm{wt}, 2}^{\mathrm{P}}, \ldots, 1 / T_{\mathrm{wt}, \mathcal{N}_{\mathrm{T}}}^{\mathrm{Q}}\right], \\
A_{\mathrm{wt}}^{\mathrm{Q}}=\operatorname{diag}\left[-1 / T_{\mathrm{wt}, 1}^{\mathrm{Q}},-1 / T_{\mathrm{wt}, 2}^{\mathrm{Q}}, \ldots,-1 / T_{\mathrm{wt}, \mathcal{N}_{\mathrm{T}}}^{\mathrm{Q}}\right], \\
B_{\mathrm{wt}}^{\mathrm{Q}}=\operatorname{diag}\left[1 / T_{\mathrm{wt}, 1}^{\mathrm{Q}}, 1 / T_{\mathrm{wt}, 2}^{\mathrm{Q}}, \ldots, 1 / T_{\mathrm{wt}, \mathcal{N}_{\mathrm{T}}}^{\mathrm{Q}}\right],
\end{gathered}
$$

Then, the discrete state-space model with sampling time $\Delta T_{\mathrm{p}}$ can be derived from the continuous model, which is,

$$
\Delta x(k+1)=\boldsymbol{A}_{\mathrm{d}} \Delta x(k)+\boldsymbol{B}_{\mathrm{d}} \Delta u(k),
$$

where

$$
\boldsymbol{A}_{\mathrm{d}}=e^{A \Delta T_{\mathrm{p}}}, \boldsymbol{B}_{\mathrm{d}}=\int_{0}^{\Delta T_{\mathrm{p}}} e^{A t} B d t
$$

\subsection{Sensitivity coefficient calculation}

Sensitivity coefficients are used to estimate the changes of objective variables in a dynamic process of the wind farm control system. In order to improve the computation efficiency, an analytical computation method for calculating the sensitivity coefficients was developed in [24], [29]. Since the capacity of each wind farm is much less than the total capacity of WFC, we assume the POC bus is the slack bus in the lower-level control. $\mathcal{S}$ and $\mathcal{N}$ denote the sets of the slack bus and the buses with PQ injections, respectively. The link between bus voltages and power injections is,

$$
\underline{S}_{i}=\underline{V}_{i} \sum_{j \in \mathcal{S} \cup \mathcal{N}} \bar{Y}_{\text {bus }, i j} \bar{V}_{j}
$$

Where $\underline{V}_{i}$, and $\underline{S}_{i}$ are the conjugates of $\bar{V}_{i}, \bar{S}_{i}$, and $\bar{Y}_{\text {bus }, i j}$ is the admittance matrix. The $\partial \underline{S}_{i} / \partial P_{l}$, and $\partial \underline{S}_{i} / \partial Q_{l}$ satisfy the following equations:

$$
\begin{aligned}
\frac{\partial \underline{S}_{i}}{\partial P_{l}} & =\frac{(P-j Q)}{\partial P_{l}}=\frac{\partial \underline{V}_{i}}{\partial P_{l}} \sum_{j \in \mathcal{S} \cup \mathcal{N}} \bar{Y}_{\text {bus }, i j} \bar{V}_{j} \\
& +\underline{V}_{i} \sum_{j \in \mathcal{N}} \bar{Y}_{\text {bus }, i j} \frac{\partial \bar{V}_{j}}{\partial P_{l}}= \begin{cases}1, & \text { for } i=l \\
0, & \text { for } i \neq l\end{cases}
\end{aligned}
$$




$$
\begin{aligned}
\frac{\partial \underline{S}_{i}}{\partial Q_{l}} & =\frac{(P-j Q)}{\partial Q_{l}}=\frac{\partial \underline{V}_{i}}{\partial Q_{l}} \sum_{j \in \mathcal{S} \cup \mathcal{N}} \bar{Y}_{\text {bus }, i j} \bar{V}_{j} \\
& +\underline{V}_{i} \sum_{j \in \mathcal{N}} \bar{Y}_{\text {bus }, i j} \frac{\partial \bar{V}_{j}}{\partial Q_{l}}= \begin{cases}-j 1, & \text { for } i=l \\
0, & \text { for } i \neq l\end{cases}
\end{aligned}
$$

According to the theorem in [24] and [29], (35) and (36) have a unique solution for radial networks. The voltage magnitude and phase angle sensitivity can be computed by,

$$
\begin{gathered}
\frac{\partial V_{i}}{\partial P_{l}}=\frac{1}{\partial V_{i}} \operatorname{Re}\left(\underline{V}_{i} \frac{\partial \bar{V}_{i}}{\partial P_{l}}\right), \frac{\partial \theta_{i}}{\partial P_{l}}=\frac{1}{\partial V_{i}^{2}} \operatorname{Im}\left(\underline{V}_{i} \frac{\partial \bar{V}_{i}}{\partial P_{l}}\right) \\
\frac{\partial V_{i}}{\partial Q_{l}}=\frac{1}{\partial V_{i}} \operatorname{Re}\left(\underline{V}_{i} \frac{\partial \bar{V}_{i}}{\partial Q_{l}}\right), \frac{\partial \theta_{i}}{\partial Q_{l}}=\frac{1}{\partial V_{i}^{2}} \operatorname{Im}\left(\underline{V}_{i} \frac{\partial \bar{V}_{i}}{\partial Q_{l}}\right)
\end{gathered}
$$

For a bus $i \in \mathcal{N}$, the partial derivatives with respect to voltage magnitude $V_{k}$ of a slack bus $k \in \mathcal{S}$ are derived by,

$$
-\underline{V}_{i} \bar{Y}_{\text {bus }, i k} e^{j \theta_{k}}=\underline{W}_{i k} \sum_{\mathcal{S} \cup \mathcal{N}} \bar{Y}_{\text {bus }, i j} \bar{V}_{j}+\underline{V}_{i} \sum_{j \in \mathcal{N}} \bar{Y}_{\text {bus }, i j} \bar{W}_{j k}
$$

where

$$
\bar{W}_{i k}=\frac{\partial \bar{V}_{i}}{\partial \bar{V}_{k}}=\left(\frac{1}{V_{i}} \frac{\partial V_{i}}{\partial V_{k}}+j \frac{\partial \theta_{i}}{\partial V_{k}}\right)
$$

According to the theorem in [24] and [29], it also has a unique solution. By solving it, the sensitivity coefficient with respect to the slack bus voltage magnitude is calculated by,

$$
\frac{\partial V_{i}}{\partial V_{k}}=V_{i} \operatorname{Re}\left(\frac{\bar{W}_{i k}}{\bar{V}_{i}}\right), \frac{\partial \theta_{i}}{\partial V_{k}}=\operatorname{Im}\left(\frac{\bar{W}_{i k}}{\bar{V}_{i}}\right)
$$

\subsection{MPC formulation for active and reactive power control of wind farm}

The MPC is a widely used optimal control method. The control input is obtained by solving a discretetime optimal control problem over a given horizon. An optimal control input sequence is generated and only the first control in the sequence is applied [30]. In this section, there are $N_{\mathrm{c}}$ control periods in a prediction horizon, the control period $T_{\mathrm{c}}$ is set in seconds, and each $T_{\mathrm{c}}$ is divide into $N_{\mathrm{p}}$ prediction periods. The control actions are only changed at the beginning of the control period and maintained within the control period.

\subsubsection{Objective function}

The objective function is expressed as:

$$
\min \sum_{i=1}^{\left|\mathcal{N}_{\mathrm{T}}\right|} \sum_{k=1}^{N_{p}}\left\|\Delta V_{\mathrm{wt}}(k)\right\|_{Q_{\mathrm{V}}}^{2}+\left\|\Delta P_{\mathrm{wt}}^{\mathrm{D}}(k)\right\|_{Q_{\mathrm{D}}}^{2}
$$

where $Q_{\mathrm{V}}$ is the weighting factors for minimizing the deviation of WT terminal voltage, and $Q_{\mathrm{D}}$ is a weighting factor for minimizing the deviation of power reference to its proportional value.

The first objective is for voltage regulation. The terminal voltages of WTs are limited in a feasible range. The predictive value of $\Delta V_{\mathrm{wt}}$ can be calculated by,

$$
\Delta V_{\mathrm{wt}}(k)=V_{\mathrm{wt}}^{0}+\frac{\partial V_{\mathrm{wt}}}{\partial P_{\mathrm{wt}}} \Delta P(k)+\frac{\partial V_{\mathrm{wt}}}{\partial Q_{\mathrm{wt}}} \Delta Q(k)-V_{\mathrm{wt}}^{\mathrm{rated}}
$$

The second objective is minimizing the deviation of power reference to its fair power sharing. The predictive value of $\Delta P_{\mathrm{wt}}^{\mathrm{D}}$ can be calculated by,

$$
\Delta P_{\mathrm{wt}}^{\mathrm{D}}(k)=P_{\mathrm{wt}}^{0}+\Delta P_{\mathrm{wt}}(k)-P_{i}^{*}
$$

where $P_{i}^{*}$ is the fair power sharing reference for the $i$ th WT according to its available power. 


\subsection{Constraints}

For a wind farm, the active and reactive power output should track the references from the upper level control,

$$
\begin{aligned}
& \sum_{j=1}^{\left|\mathcal{N}_{\mathrm{T}}^{(i)}\right|} P_{\mathrm{wt}, j}^{\mathrm{ref}}=P_{\mathrm{WF}, i}^{\mathrm{ref}}, \quad \forall i \in \mathcal{N}_{\mathrm{F}} \\
& \sum_{j=1}^{\left|\mathcal{N}_{\mathrm{T}}^{(i)}\right|} Q_{\mathrm{wt}, j}^{\mathrm{ref}}=Q_{\mathrm{WF}, i}^{\mathrm{ref}}, \quad \forall i \in \mathcal{N}_{\mathrm{F}}
\end{aligned}
$$

For a WT, the available active and reactive power limits are as follows,

$$
\begin{gathered}
0 \leq P_{\mathrm{wt}, j}^{\mathrm{ref}} \leq P_{\mathrm{wt}, j}^{\mathrm{avi}}, \forall j \in \mathcal{N}_{\mathrm{T}}^{(i)} \\
-Q_{\mathrm{wt}, j}^{\mathrm{avi}} \leq Q_{\mathrm{wt}, j}^{\mathrm{ref}} \leq Q_{\mathrm{wt}, j}^{\mathrm{avi}}, \forall j \in \mathcal{N}_{\mathrm{T}}^{(i)}
\end{gathered}
$$

It is better to set voltage limits in every prediction steps to make sure the WT terminal voltages do not exceed thresholds, which is as follows,

$$
V_{\mathrm{wt}}^{\min } \leq V_{\mathrm{wt}, j} \leq V_{\mathrm{wt}}^{\max }, \forall j \in \mathcal{N}_{\mathrm{T}}^{(i)}
$$

where $V_{\mathrm{wt}}^{\max }$ and $V_{\mathrm{wt}}^{\min }$ are the maximal and minimal thresholds of WT terminal voltages. The MPC problem can be reformulated as a standard Quadratic Programming (QP) problem and can be efficiently solved by commercial solvers. In this paper, the MATLAB quadprog function was used to solve the QP problem.

\section{Case Study}

\subsection{Test system}

The WFC in Fig. 1 is used to demonstrate the performance of the proposed bi-level DARPC scheme. In order to improve the simulation efficiency, only one of the eight wind farms is modelled with detailed WT models (Wind Farm 1). The remaining 7 wind farms are equivalent to one WT. The system parameters are shown in Table. 1. The wind field model considering turbulences and wake effects was generated from SimWindFarm, a toolbox for dynamic wind farm model, simulation and control. The wind field model is used to get available wind power for each wind farm.

In the test system, Wind Farm 1 and Wind Farm 2 are defined as the leaders. For the upper-level control, the average available active and reactive power are calculated and updated every $5 \mathrm{~s}$. For the lower-level control, the control period $T_{\mathrm{c}}$, prediction horizon $T_{\mathrm{p}}$ and sampling period of prediction $\Delta T_{\mathrm{p}}$ are set as 1 $\mathrm{s}, 5 \mathrm{~s}$, and $0.1 \mathrm{~s}$, respectively. In order to examine the performance of the proposed control method, the simulation results are compared with the ones based on the conventional centralized proportion distribution (PD) scheme and the centralized active and reactive power control (CARPC). The CARPC is described in Appendix.

\subsection{Control performance under normal operation}

The performance of the proposed control scheme under normal operation is tested in this subsection. The total simulation time is set as $600 \mathrm{~s}$. Fig. 6 shows the total available wind power, the active power dispatch command from the TSO. The total available wind power fluctuates between 550 to $650 \mathrm{MW}$ during $t=0-250 \mathrm{~s}$. During $t=250-380 \mathrm{~s}$, the available wind power gradually rises. After $t=380 \mathrm{~s}$, the total available wind power gradually decreases. The dispatch command is set as $400 \mathrm{MW}$ during $t=0-250$ $\mathrm{s}$ and then increases within the ramp limit during $t=250-360 \mathrm{~s}$. After $t=360 \mathrm{~s}$, it is set as $700 \mathrm{MW}$. At around $400 \mathrm{~s}$, the available wind power is less than the dispatch command, based on distributed control method in this paper, the WFC operates in the maximum power point tracking mode (MPPT). The reactive power reference generated by the leader controller is shown in Fig. 7. It is updated every 5 seconds. The WFC absorbs reactive power from $t=0-250 \mathrm{~s}$, and gradually decreases after $250 \mathrm{~s}$. In the whole control 
Table 1. ELECTRICAL SYSTEM PARAMETERS

\begin{tabular}{ll}
\hline 33kV Cable & $R_{33 \mathrm{kV}}=0.078 \Omega / \mathrm{km}, L_{33 \mathrm{kV}}=0.3915 \mathrm{mH} / \mathrm{km}, C_{33 \mathrm{kV}}=0.13 \mu \mathrm{F} / \mathrm{km}$ \\
$155 \mathrm{kV}$ Cable & $R_{155 \mathrm{kV}}=0.0328 \Omega / \mathrm{km}, L_{155 \mathrm{kV}}=0.47 \mathrm{mH} / \mathrm{km}, C_{155 \mathrm{kV}}=0.11 \mu \mathrm{F} / \mathrm{km}$ \\
$380 \mathrm{kV}$ Cable & $R_{380 \mathrm{kV}}=0.018 \Omega / \mathrm{km}, L_{380 \mathrm{kV}}=0.47 \mathrm{mH} / \mathrm{km}, C_{155 \mathrm{kV}}=0.13 \mu \mathrm{F} / \mathrm{km}$ \\
$0.9 \mathrm{kV} / 33 \mathrm{kV}$ Transformer & $S_{0.9 / 33 \mathrm{kV}}=6.25 \mathrm{MVA}, R_{0.9 / 33 \mathrm{kV}}=0.008 \mathrm{p} . \mathrm{u} ., L_{0.9 / 33 \mathrm{kV}}=0.06 \mathrm{p} . \mathrm{u}$. \\
$33 \mathrm{kV} / 155 \mathrm{kV}$ Transformer & $S_{33 / 155 \mathrm{kV}}=100 \mathrm{MVA}, R_{33 / 155 \mathrm{kV}}=0.002$ p.u., $L_{33 / 155 \mathrm{kV}}=0.006 \mathrm{p} . \mathrm{u}$. \\
OLTC & $S_{\mathrm{OLTC}}=800 \mathrm{MVA}, R_{\mathrm{OLTC}}=0.005$ p.u., $L_{\mathrm{OLTC}}=0.06$ p.u. \\
WFC rated capacity & $S_{\mathrm{WFC}}=800 \mathrm{MVA}$ \\
Wind farm rated capacity & $S_{\mathrm{WF}}=100 \mathrm{MVA}$ \\
WT rated capacity & $S_{\mathrm{WT}}=6.25 \mathrm{MVA}$ \\
Constant gain $\mu_{\mathrm{P}}$ & 0.3 \\
Constant gain $\varepsilon_{\mathrm{P}}$ & 0.5 \\
Constant gain $\mu_{\mathrm{Q}}$ & 0.3 \\
Constant gain $\varepsilon_{\mathrm{Q}}$ & 0.5 \\
Time constant $T_{\mathrm{wt}}^{\mathrm{Q}}$ & $1 \mathrm{~s}$ \\
Time constant $T_{\mathrm{wt}}^{\mathrm{P}}$ & $1 \mathrm{~s}$ \\
Weight $Q_{\mathrm{V}}$ & 0.25 \\
Weight $Q_{\mathrm{d}}$ & 0.0125 \\
\hline
\end{tabular}

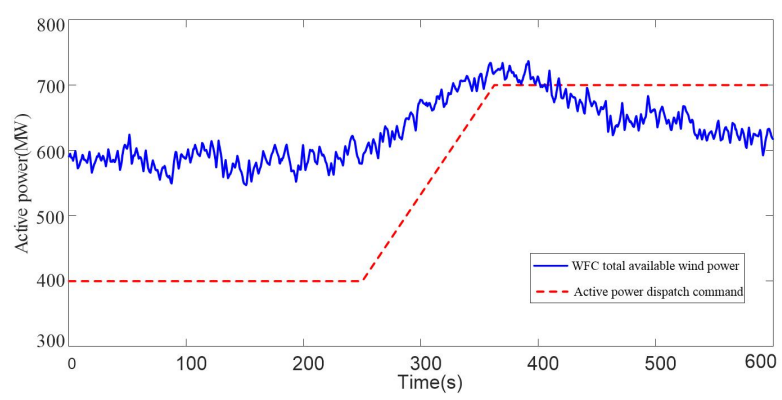

Fig. 6. The total available wind power and the dispatch command.

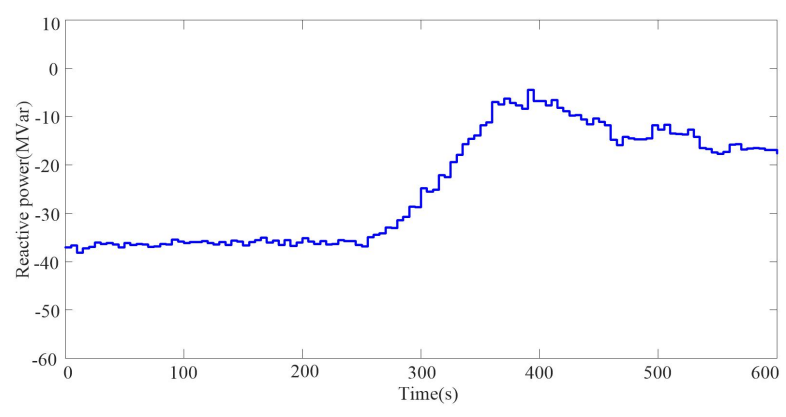

Fig. 7. Reactive power reference.

period, the WFC absorbs reactive power to control POC voltage fluctuation caused by the increasing wind power of the WFC. 
The average available wind power and reactive power estimated by each wind farm from $200 \mathrm{~s}$ to $300 \mathrm{~s}$ are shown in Fig. 8 and 10 , respectively. The estimated average available wind power varies with the realtime available wind power of each wind farm. The estimated average available reactive power varies with the available wind power. When the average available wind power increases, the average available reactive power decreases. The estimated average available active and reactive power estimated by each wind farm can converge to the actual value, implying good convergence. The utilization ratio of wind power and reactive power from $200 \mathrm{~s}$ to $300 \mathrm{~s}$ is shown in Figs. 9 and 11, respectively. It can be seen that all wind farms have consistent utilization ratio, indicating that the proposed upper-level controller can effectively regulate the active and reactive power outputs of wind farms to maintain fair power sharing. For the utilization ratio, the leader controller firstly reaches newly updated desired utilization ratio and then followers starts to track the desired utilization ratio. All calculation can be done in $1.5 \mathrm{~s}$ at every control period, which is fast enough for the real-time lower-level wind farm control since it is generally designed in seconds.

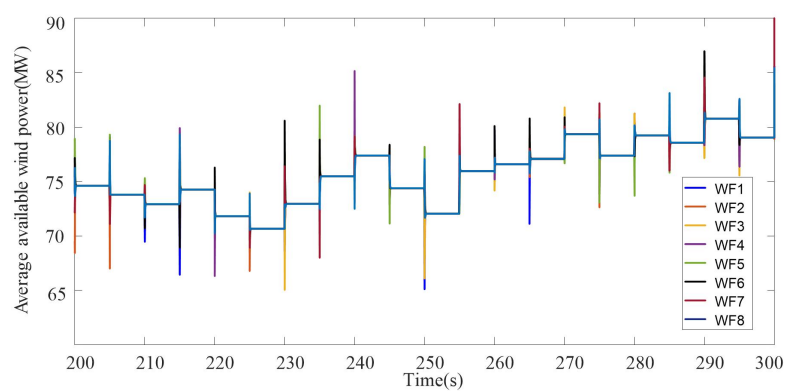

Fig. 8. Estimated average available wind power of WFC.

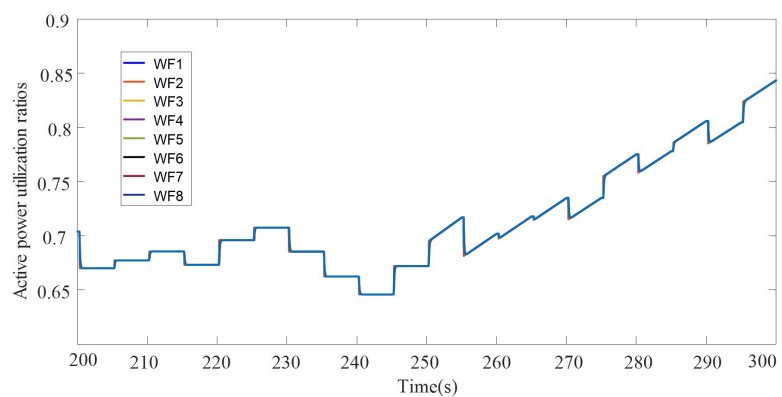

Fig. 9. Utilization of each wind farm.

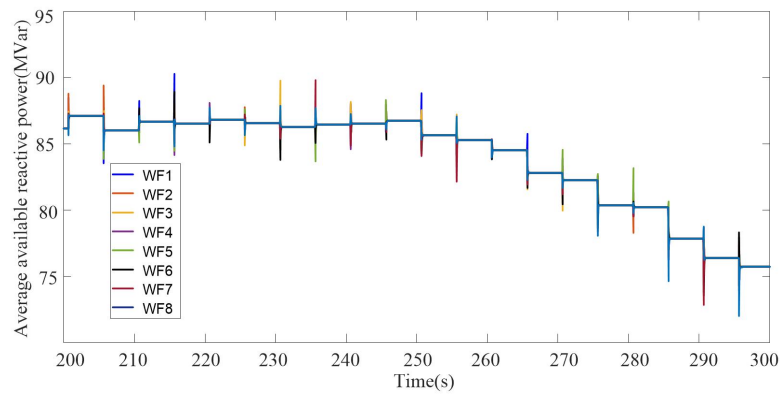

Fig. 10. Estimated average available reactive power of WFC. 


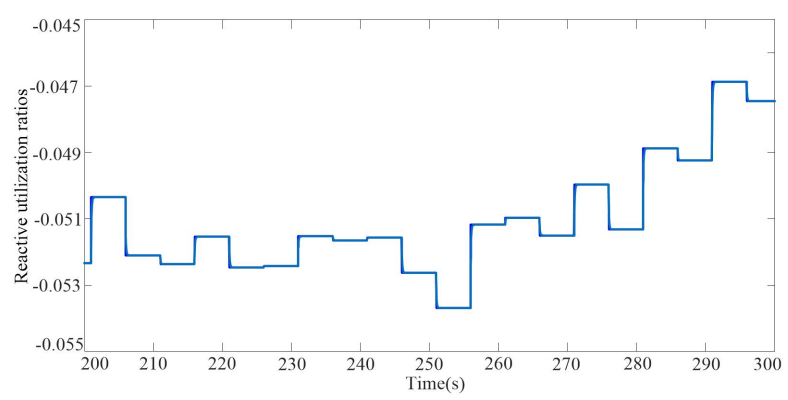

Fig. 11. Reactive power utilization of each wind farm..

The comparison of the DARPC, the centralized PD control scheme performance, and the CARPC method is shown in Figs. 12-14. The POC voltage is shown in Fig. 12. It can be seen that the distributed control scheme has similar control performance as the centralized PD control scheme and they can both effectively control the POC voltage at around 1 p.u. The performance with the CARPC is different from the DARPC from 250 - $600 \mathrm{~s}$. It is because that, in the CARPC method, the objective of the WFC controller is keeping all bus voltage within feasible range (see Appendix). In the DARPC methods, the objective of the upper-level control is controlling the POC voltage only. The WFC output active and reactive power is shown in Figs. 13-14. For the PD control and DARPC, they can both effectively regulate the active power output of the WFC to track the power reference set by the TSO, and track the reactive power reference generated by leaders. For the CARPC, the reactive power output is different from the DARPC. The active and reactive output of wind farm 4 are shown in Figs 15-16. The active power output is similar with the three kinds of control methods. The reactive power output with the CARPC method is different from the PD control and DARPC. However, compared with the centralized control, the distributed control scheme eliminates the central supervisor controller and largely reduces the communication cost, implying better cost benefits and robustness. Moreover, since each wind farm only exchanges information with its neighbors, the WFC has good plug-and-play capability, providing the convenience to extend the WFC.

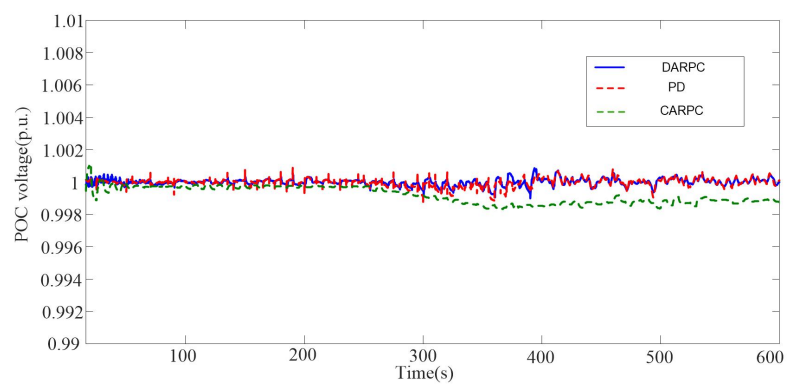

Fig. 12. Poc voltage.

The performance of the lower-level DARPC is shown in Figs. 17-19, which coordinates active and reactive power of all WTs inside a wind farm. Similarly, the centralized PD control and CARPC are simulated to compare with the DARPC. WT 5 is selected as the representative WT to illustrate the results. It can be seen that, the voltage deviation with the DARPC is much smaller than that with the PD control scheme, especially during $t=400-600 \mathrm{~s}$, all WTs operate in the maximum power tracking mode. The voltage deviation with the CARPC is slightly smaller than with the DARPC. The output reactive power with DARPC is quit different from the PD method and slightly different from the CARPC. The output active power with the DARPC is similar as with the PD method and the CARPC. Comparably, the performance of the DARPC is better. 


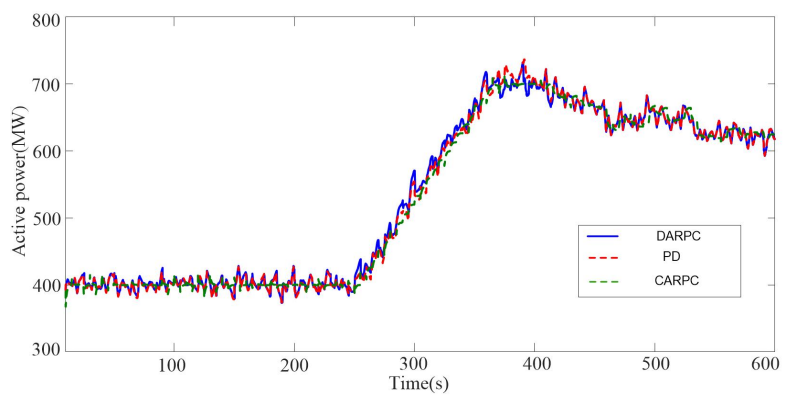

Fig. 13. WFC output active power.

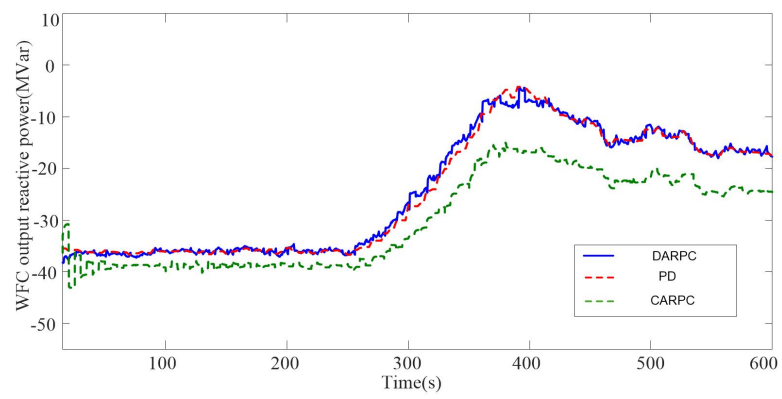

Fig. 14. WFC output reactive power.

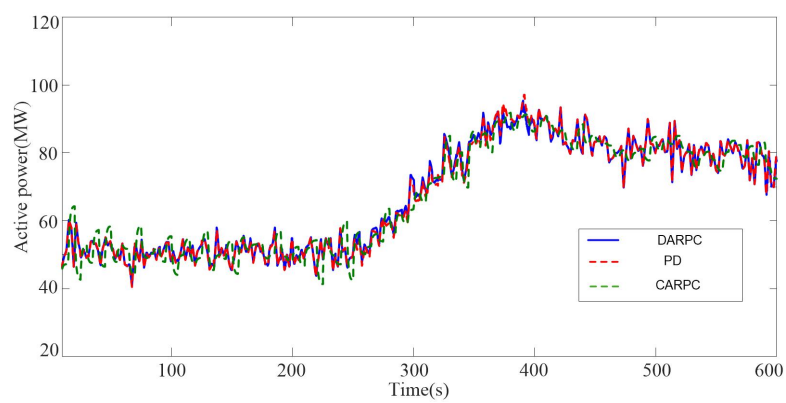

Fig. 15. WF 4 output active power.

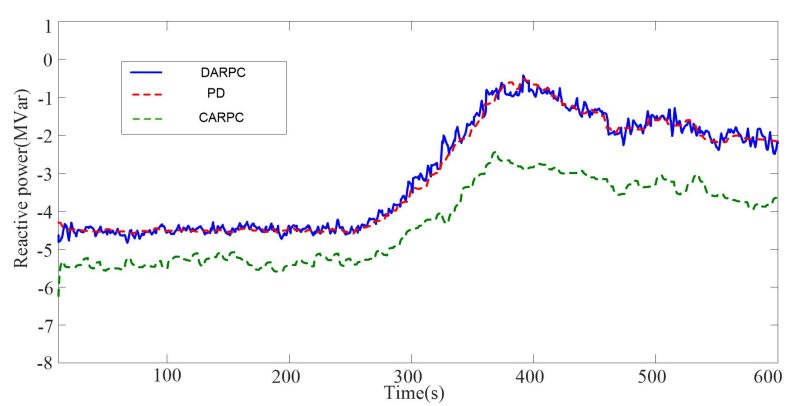

Fig. 16. WF 4 output reactive power.

\subsection{Operation With a grid disturbance}

In this section, a disturbance of the external grid is considered. At the beginning, the WFC operate in a normal condition. At $62 \mathrm{~s}$, the external grid voltage drops to $0.9 \mathrm{p}$.u, and it is cleared at $t=122 \mathrm{~s}$. The 


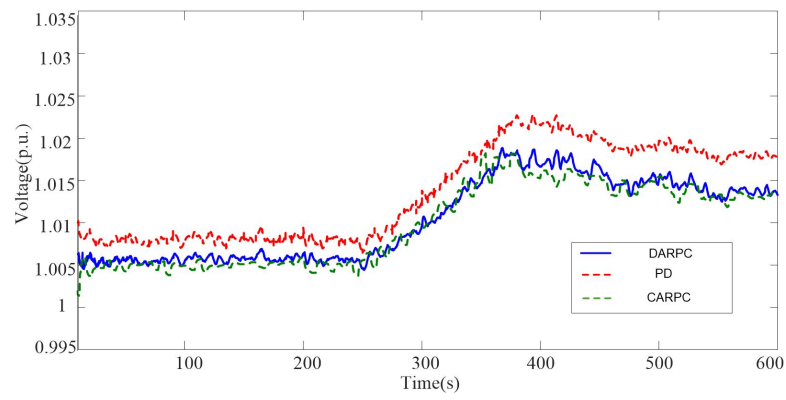

Fig. 17. WT 5 terminal voltage.

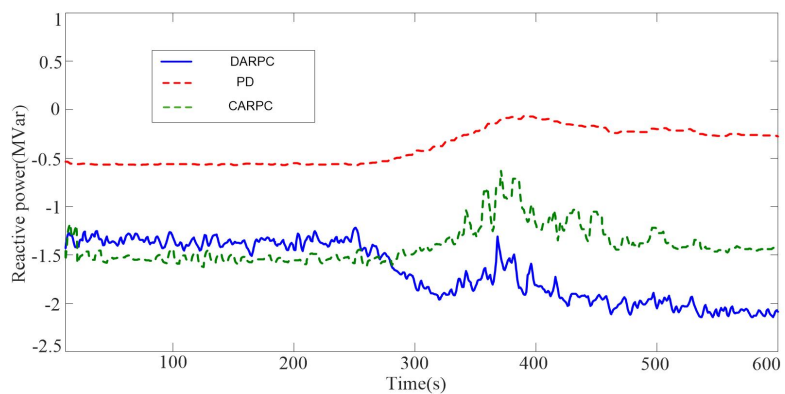

Fig. 18. WT 5 output reactive power.

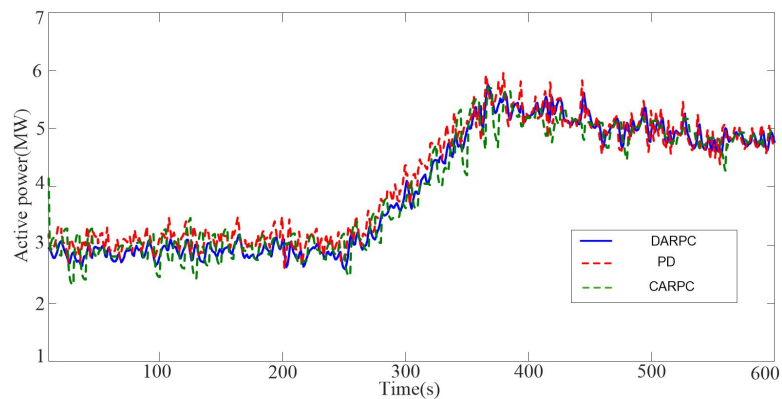

Fig. 19. WT 5 output active power.

simulation time is $180 \mathrm{~s}$. The simulation results are illustrated in Figs. 20-23.

The POC voltage is shown in Fig. 22. At $62 \mathrm{~s}$, the external grid disturbance results in a sudden decrease of $V_{P O C}$. The leader controller updates reactive power reference at $65 \mathrm{~s}$ [see Fig. 21], the WFC provides reactive power to recover the POC voltage. At the same time, the OLTC begin to change tap [see Fig. 20], after $2.5 \mathrm{~s}$, the action is done. From Fig. 20, it can be seen, the OLTC change 2 taps during the disturbance period. It can be seen, at around $66 \mathrm{~s}$, the voltage has the first step increase due to the WFC reactive power support to recover voltage. At around $68 \mathrm{~s}$, the voltage has the second step increase due to the OLTC action. Since the leader controllers consider the OLTC action, the incremental reactive power at $71 \mathrm{~s}$ is much smaller than incremental reactive power at $66 \mathrm{~s}$. At $73 \mathrm{~s}$, the POC voltage recovers to 1 p.u.. The transformer HV side voltage is shown in Fig. 23. The WFC can provide reactive power to support grid when there is a disturbance in the grid. At $122 \mathrm{~s}$, the external grid voltage recovers, the WFC switches back to the normal operation. Figs. $20-23$ show that the distributed control can not only regulate the POC voltages to be in feasible range during the grid voltage disturbance, but also provide reactive power support to the external grid. 


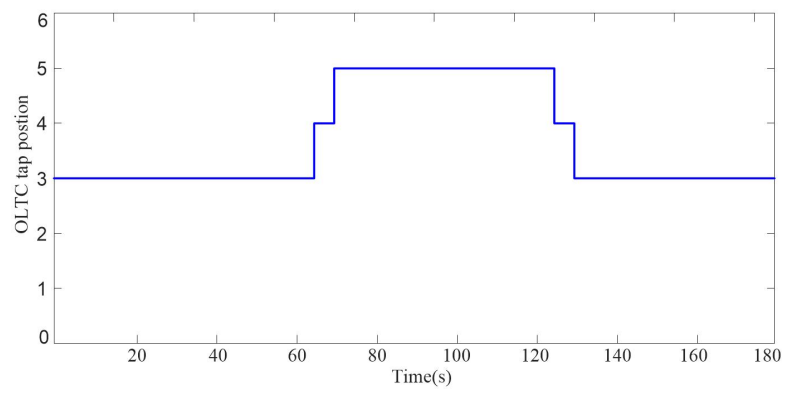

Fig. 20. OLTC tap position.

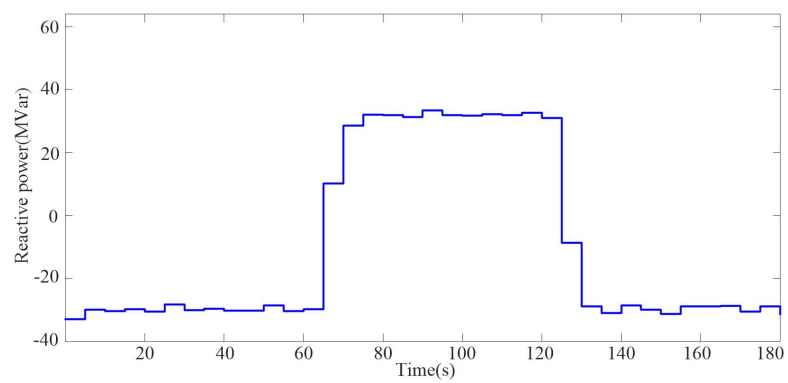

Fig. 21. Reactive reference for WFC.

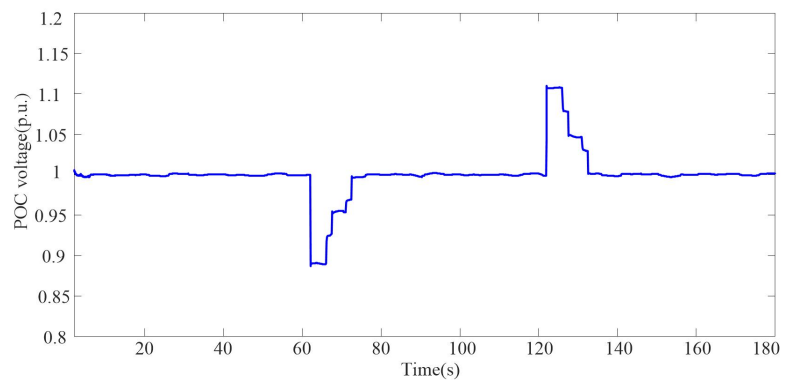

Fig. 22. POC voltage.

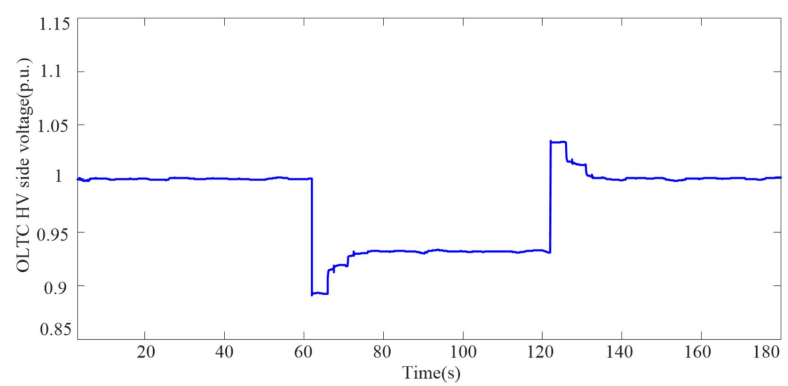

Fig. 23. OLTC HV side voltage. 


\section{Conclusion}

In this paper, a bi-level decentralized active and reactive power control scheme is proposed for the large-scale WFC. In the upper-level control, the consensus protocol method is used to estimate the available active and reactive power of the WFC, and get the utilization ratios for each wind farm controller. The wind farm controller only communicates with neighboring wind farm controllers. The leader control generate the reactive power reference while considering the OLTC action. In the lower level control, the WTs in the wind farm are controlled in a centralized manner based on the MPC. Case studies show the WFC can control the POC voltage in a feasible range while accurately tracking the active power dispatch command, and also minimizing the voltage deviation of WTs inside the wind farm. Moreover, during the grid voltage disturbance, the distributed control can not only regulate the POC voltage to be in the feasible range, but also provide reactive power support to the external grid. The proposed bi-level DARPC is suitable for realtime control of large-scale WFCs. It does not require a central WFC controller, simplifies communication networks of large-scale WFCs, and effectively minimize voltage deviation of the POC and WT terminals.

\section{APPENDIX}

\section{CARPC Method}

In the centralized control method, the incremental voltage of the $i$ th bus voltage can be expressed as,

$$
\Delta V_{i}=\frac{\partial V}{\partial Q} \Delta Q+\frac{\partial V}{\partial P} \Delta P
$$

Denote $\mathcal{N}_{\mathrm{w}}$ as the set of WTs inside the WFC, $\mathcal{N}_{\mathrm{D}}$ as the set of PQ nodes inside the WFC, the incremental voltages of the WFC can be expressed as a matrix form:

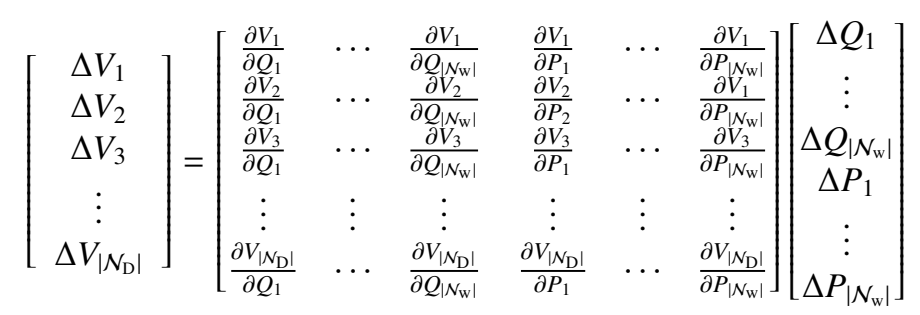

Normally, the dispatch algorithm for the WFC is based on a proportional distribution of the available active power. The power reference of the $i$ th WT inside the WFC is calculated by,

$$
P_{\mathrm{w}, i}^{\mathrm{PD}}=\alpha_{\mathrm{pd}} P_{\mathrm{WFC}}^{\mathrm{ref}}, \quad \alpha_{\mathrm{pd}}=\frac{P_{\mathrm{w}, i}^{\mathrm{avi}}}{P_{\mathrm{WFC}}^{\mathrm{avi}}}
$$

The objective function is expressed as,

$$
\begin{aligned}
\min & \sum_{k=1}^{N_{\mathrm{P}}}\left\|\Delta V_{\mathrm{POC}}+V_{\mathrm{POC}}^{0}-V_{\mathrm{POC}}^{\text {rated }}\right\|_{Q_{\mathrm{C}}}^{2}+\sum_{k=1}^{N_{\mathrm{P}}} \sum_{i=1, i \neq \mathrm{POC}}^{\left|\mathcal{N}_{\mathrm{D}}\right|}\left\|\Delta V_{i}+V_{i}^{0}-V_{i}^{\text {rated }}\right\|_{Q_{\mathrm{V}}}^{2} \\
& \sum_{k=1}^{N_{\mathrm{P}}} \sum_{i=1}^{\left|\mathcal{N}_{\mathrm{w}}\right|}\left\|\Delta P_{\mathrm{wt}, i}+P_{\mathrm{wt}, i}^{0}-P_{\mathrm{wt}, i}^{\mathrm{PD}}\right\|_{Q_{\mathrm{P}}}^{2}
\end{aligned}
$$

For the WFC, the active power output should track the dispatch command,

$$
\sum_{i=1}^{\left|\mathcal{N}_{\mathrm{w}}\right|} P_{\mathrm{wt}, i}^{\mathrm{ref}}=P_{\mathrm{WFC}}^{\mathrm{ref}}, \quad \forall i \in \mathcal{N}_{\mathrm{w}}
$$

For a WT, the available active and reactive power limits are as follows,

$$
\begin{gathered}
0 \leq P_{\mathrm{wt}, i}^{\mathrm{ref}} \leq P_{\mathrm{wt}, i}^{\mathrm{avi}}, \forall i \in \mathcal{N}_{\mathrm{w}} \\
-Q_{\mathrm{wt}, i}^{\mathrm{avi}} \leq Q_{\mathrm{wt}, i}^{\mathrm{ref}} \leq Q_{\mathrm{wt}, i}^{\mathrm{avi}}, \forall i \in \mathcal{N}_{\mathrm{w}}
\end{gathered}
$$




\section{References}

[1] Wind energy scenarios for 2030, European Wind Energy Association. [Online]. Available:https://windeurope.org/fileadmin/files/library/publicatio ns/reports/EWEA-Wind-energy-scenarios-2030.pdf

[2] Energy Policy in Denmark, Danish Energy Agency, Dec. 2012.

[3] P. Rodrguez, A. Timbus, R. Teodorescu, M. Liserre, F. Blaabjerg, Reactive power control for improving wind turbine system behavior under grid faults, IEEE Trans. on Power Elect. 24(7)(2009)1798-1801.

[4] R. Perveen, N. Kishor, S .R. Mohanty, Off-shore wind farm development: Present status and challenges, Renew. and Sustain. Energy Rev.(29)(2014)780-792.

[5] M. Wolff, R. Mackensen, G. Füller, B. Lange, K. Rohrig,Advanced operating control for wind farm clusters,in:Sixth International Workshop on Large-Scale Integration of Wind Power and Transmission Networks for Offshore Wind Farms. 2006, pp.188-195

[6] T. Neumann, C. Feltes, and I. Erlich, Response of DFG-basedwind farms operating on weak grids to voltage sags,in:Proc. Power Eng. Soc. Gen. Meet., 2011, pp.16.

[7] M. Firouzi, G. B. Gharehpetian, B. Mozafari, Power Flow Control and Short Circuit Current Limitation of Wind Farms Using Unified Inter-Phase Power Controller, IEEE Trans. Power Del. 32(1)(2017)62-71.

[8] P. Sørensen, A. D. Hansen, F. Iov, F. Blaabjerg, M. H. Donovan, Wind farm models and control strategies. Aug. 2005.

[9] Z. Lubosny, J. W. Bialek, Supervisory control of a wind farm, IEEE Trans. Power Syst.. 22(3)(2007),985-994

[10] X. Guan and G. M. vander Molen, Control strategy review and specification (Part 1), 2009 [Online].

[11] D. Li, S. Wang, W. Lei, Method for wind farm cluster active power optimal dispatch under restricted output condition. Electric Utility Deregulation and Restructuring and Power Technologies (DRPT), in:2015 5th International Conference on. IEEE, 2015, pp.1981-1986

[12] H. Zhao, Q. Wu, Q. Guo, Optimal active power control of a wind farm equipped with energy storage system based on distributed model predictive control, IET Gen., Trans. and Distri.. 10(3)(2016)669-677.

[13] T. Kaneko, T. Senjyu, A. Yona, M. Datta, T. Funabashi, and C. H. Kim, Output power coordination control for wind farm in small power system, in:14th Int. Conf. Intel. Syst. Appl. Power Syst. (ISAP 2007), 2007, pp.5156.

[14] A. D. Hansen, P. E. SØrensen, F. Iov, F. Blaabjerg, Centralised power control of wind farm with doubly fed induction generators, Renew. Energy,31(7)(2006)935951.

[15] B. Zhang, P. Hou, W. Hu, M. Soltani, C. Chen, Z. Chen, A reactive power dispatch strategy with loss minimization for a DFIGbased wind farm. IEEE Trans. on Sust. Energy.7(3)(2016)914-923.

[16] N. R. Ullah, K. Bhattacharya, T. Thiringer, Wind farms as reactive power ancillary service providerstechnical and economic issues. IEEE Trans. on Energy Conv.24(3)(2009)661-672.

[17] G. Tapia, A. Tapia, J. X. Ostolaza, Proportionalintegral regulator-based approach to wind farm reactive power management for secondary voltage control. IEEE Trans.on Energy Conv.22(2)(2007)488-498.

[18] B .R. Karthikeya, R. J. Schutt, Overview of wind park control strategies. IEEE Trans. on Sust. Energy,5(2)(2014),416-422

[19] J. Martí nez, P. C. Kjær, P. Rodriguez, and R. Teodorescu, Comparison of two voltage control strategies for a wind power plant.in: Proc. IEEE/PES Power Syst. Conf. Expo. (PSCE), 2011, pp. 19.

[20] J. N. Sakamuri, Z.H. Rather,J. Rime,M. Altin,O. Goksu,N. A. Cutululis, Coordinated voltage control in offshore HVDC connected cluster of wind power plants, IEEE Trans. Sustain. Energy.7(4)(2016)1592-1601.

[21] Q. Guo, H. Sun, B. Wang, B. Zhang, W. Wu and L. Tang, Hierarchical automatic voltage control for integration of large-scale wind power: Design and implementation.Electr. Power Syst. Res.,120(3)(2015)234- 241.

[22] H. Zhao, Q. Wu, Q. Guo,H. Sun, S. Huang, Y. Xue, Coordinated voltage control of a wind farm based on model predictive control. IEEE Trans. on Sust. Energy,7(4)(2016)1440-1451.

[23] Y. Guo, H.Gao, Q. Wu, H. Zhao, J,Østergaard, Coordinated voltage control scheme for VSC-HVDC connected wind power plants. IET Renew. Power Gener.12(2)(2017)198-206

[24] Y. Guo, H. Gao, Q. Wu, H. Zhao, J,Østergaard, Enhanced Voltage Control of VSC-HVDC Connected Offshore Wind Farms Based on Model Predictive Control.IEEE Trans. on Sust. Energy, 9(1)(2018)474-487.

[25] R. Olfati-Saber, R. M. Murray, Consensus problems in networks of agents with switching topology and time-delays. IEEE Trans. on automatic contr, 49(9) (2004)1520-1533.

[26] K. E. Muljadi, C. P. Butterfield, A. Ellis, Equivalencing the collector system of a large wind power plant.in:Power Engineering Society General Meeting, 2006.

[27] M. Muttaqi, A. D. Le, M. Negnevitsky, G. Ledwich, Acoordinated voltage control approach for coordination of OLTC, voltage regulator, and DG to regulate voltage in a distribution feeder,IEEE Trans. Ind. Appl.,51(2)(2015)12391248

[28] M. C. Alexiadis, P. S. Dokopoulos, H. S. Sahsamanoglou, Wind speed and power forecasting based on spatial correlation models. IEEE Trans. on Energy Conver., 14(3) (1999) 836-842.

[29] K. Christakou, J. LeBoudec, M. Paolone, D. C. Tomozei, Efficient computation of sensitivity coefficients of node voltages and line currents in unbalanced radial electrical distribution networks,IEEE Trans. Smart Grid,4(2)(2013)41750.

[30] E. Camponogara, D. Jia, B. H. Krogh, Distributed model predictive control. IEEE Control Syst.22(1)(2002)44-52 